\title{
Behavior of a Free Quantum Particle in the Poincaré Upper Half-Plane Geometry
}

\author{
Parham Dehghani* and S. Habib Mazharimousavi ${ }^{\dagger}$ \\ Department of Physics, Faculty of Arts and Sciences, \\ Eastern Mediterranean University, Famagusta, \\ North Cyprus via Mersin 10, Turkey \\ S. Danial Forghani ${ }^{\ddagger}$ \\ Faculty of Engineering, Final International University, \\ Kyrenia, North Cyprus via Mersin 10, Turkey
}

(Dated: July 2, 2020)

\begin{abstract}
Inspired by the recent work of Filho et al. [11], a Hermitian momentum operator is introduced in a general curved space with diagonal metric. The modified Hamiltonian associated with this new momentum is calculated and discussed. Furthermore, granting the validity of the Heisenberg equation in a curved space, the Ehrenfest theorem is generalized and interpreted with the new position-dependent differential operator in a curved space. The modified Hamiltonian leads to a modified time-independent Schrodinger equation, which is solved explicitly for a free particle in the Poincare upper half-plane geometry. It is shown that a "free particle" does not behave as it is totally free due to curved background geometry.

PACS: 04.60.-m, 04.70.-s, 98.80.-k, 95.36.+x

Keywords: Poincare half-plane geometry, Quantum gravity, Hermiticity, Ehrenfest theorem, Free particle
\end{abstract}

\section{INTRODUCTION}

Quantum mechanics has been expanded and theorized in the Euclidean space. It is then an important pursuit to expand the algebraic language of quantum mechanics within the framework of a curved geometry, say the geometry of the general theory of relativity. For a long time, the combination of quantum field theory and general relativity has been a holy grail that no one has ever managed to reach. Then, any endeavor to change the existing pillars of quantum world such that its modified picture lives up to a unification with general relativity is appreciated. There are thus phenomenological research works addressing the extension of quantum mechanics on a curved geometry [1-9]. Dirac and Klein-Gordon oscillators are examined on Anti-de Sitter space which include deformed Heisenberg uncertainty principle and non-commutative momentum space [10]. In Ref. [1], quantum mechanics in curved spacetime has been studied. To do so, the author employed a spherically symmetric static spacetime with the line element

$$
d s^{2}=-f(r) d t^{2}+\frac{1}{f(r)} d r^{2}+r^{2}\left(d \theta^{2}+\sin ^{2} \theta d \phi^{2}\right),
$$

and the momentum operator is defined in the form

$$
p_{j}=\frac{-i \hbar}{\sqrt{g}} \partial_{j} \sqrt{g}
$$

\footnotetext{
* parham.dehghani@emu.edu.tr

$\dagger$ habib.mazhari@emu.edu.tr

$\ddagger$ danial.forghani@final.edu.tr
}

where $j=r, \theta, \phi$ and $g$ is the determinant of the space part of the metric tensor i.e. $g=\operatorname{det}\left(g_{i j}\right)=\frac{1}{f(r)} r^{4} \sin ^{2} \theta$. Upon this definition, the generalized Laplace operator has been extracted as

$$
\nabla^{2}=\frac{1}{\sqrt{g}} \partial_{j}\left(\sqrt{g} g^{j j} \partial_{j}\right) .
$$

On the other hand, the energy operator corresponds to the time component of the metric tensor such that

$$
E=\frac{i \hbar}{\sqrt{f}} \partial_{0} .
$$

The author used the space-Laplace operator (3) and energy operator (4) to construct the Klein-Gordon and Dirac equation for spin 0 and $\frac{1}{2}$ particles, respectively. Beside the applications of these operators mentioned in Ref. [1], one may assume the spacetime metric (1) to be of the form

$$
d s^{2}=-d t^{2}+\frac{1}{f(r)} d r^{2}+r^{2}\left(d \theta^{2}+\sin ^{2} \theta d \phi^{2}\right),
$$

which consequently implies (3) for the space-Laplace, but the energy operator (4) modifies as

$$
E=i \hbar \partial_{0} .
$$

Upon considering the time $t$ to be the proper time, one may construct the curved Schrodinger equation as of

$$
\left(-\frac{\hbar^{2}}{2 m} \nabla^{2}+V(\vec{r})\right) \Psi(r, t)=i \hbar \partial_{0} \Psi(r, t) .
$$


This is what basically has been considered in a recent paper [11] by Filho et al., where they have introduced the momentum operator of a quantum particle moving in a curved space with a diagonal metric. Such a momentum depends on the structure of the curved space, and consequently the Heisenberg uncertainty relation gets modified. Moreover, in Ref. [11] the authors have explicitly shown that the Ehrenfest theorem changes due to the curvature of the space where the particle's motion is under consideration. They have introduced a non-additive translation operator in a curved space with a diagonal metric tensor. In accordance with their approach, in a one-dimensional curved metric with line element

$$
d s^{2}=g_{x x} d x^{2}
$$

an infinitesimal translation from an initial point $x$ to a final point $x+g_{x x}^{-\frac{1}{2}} d x$, one introduces the translation operator to be

$$
T(d x)|x\rangle=\left|x+g_{x x}^{-\frac{1}{2}} d x\right\rangle .
$$

This, however, implies that

$$
T(d x)=1-i \frac{P_{x}}{\hbar} d x
$$

in which $P_{x}$ is the momentum operator in $x$ direction. In three-dimensional curved space with a diagonal metric given by

$$
d s^{2}=g_{x x} d x^{2}+g_{y y} d y^{2}+g_{z z} d z^{2}
$$

one writes

$$
\begin{aligned}
& T(d \vec{r})|x, y, z\rangle= \\
& \quad\left|x+g_{x x}^{-\frac{1}{2}} d x, y+g_{y y}^{-\frac{1}{2}} d y, z+g_{z z}^{-\frac{1}{2}} d z\right\rangle,
\end{aligned}
$$

with

$$
T(d \vec{r})=1-i \frac{\vec{P}}{\hbar} \cdot d \vec{r}
$$

Hence, Eq. (10) or (13) admits

$$
\left[x, P_{x}\right]=i \hbar g_{x x}^{-\frac{1}{2}}
$$

with similar relations for $y$ and $z$. Therefore, one has to introduce $P_{j}$ such that the corresponding commutation relation (14) holds. The first choice is to set

$$
P_{j}=-i \hbar g_{j j}^{-\frac{1}{2}} \partial_{j}
$$

where $j$ stands for $x, y$ or $z$. Here, we would like to add that $P_{j}$, as given in (15), is not the most general form of the linear momentum in $j$ direction which satisfies the corresponding relation as of (14). By adding a general gauge function such as $\psi(x, y, z)$ to $(15),(14)$ still holds. Hence, we propose

$$
P_{j}=-i \hbar g_{j j}^{-\frac{1}{2}} \partial_{j}+\psi_{j}
$$

in which $\psi_{j}=\psi_{j}(x, y, z)$ is an arbitrary gauge function, corresponding to the $j$ direction. On the other hand, $\hat{P}_{j}$ is supposed to represent the momentum operator in $j$ direction. This, however, suggests that $\hat{P}_{j}$ should be locally Hermitian. To apply the local-Hermiticity condition, we assume that the space is flat in the local coordinate system, spanned by the local Cartesian coordinates $\{x, y, z\}$, such that $\left(\partial_{j}\right)^{\dagger}=-\partial_{j}$. Considering the latter, one finds $\psi_{j}=-\frac{1}{2} i \hbar\left(g_{j j}^{-\frac{1}{2}}\right)$ and consequently the locally Hermitian momentum operator becomes

$$
P_{j}=-\frac{1}{2} i \hbar\left(g_{j j}^{-\frac{1}{2}} \partial_{j}+\partial_{j} g_{j j}^{-\frac{1}{2}}\right) .
$$

Staying in the local $\{x, y, z\}$ coordinate system and employing the corresponding local momentum operators (17), one, in principle, may write the time-independent Schrodinger equation of a quantum particle, undergoing a general time-independent potential $V(\vec{r})$, as

$$
\frac{1}{2 m}\left(P_{j}\right)^{2} \Psi(\vec{r})+V(\vec{r}) \Psi(\vec{r})=E \Psi(\vec{r}),
$$

in which $E$ and $m$ stand for the energy and the mass of the particle, respectively, while $V(\vec{r})$ is the timeindependent potential. Inserting (17) in (18), one explicitly finds

$$
\begin{aligned}
-\frac{\hbar^{2}}{2 m} \sum_{j=1}^{3}\left(g_{, j}^{j j} \partial_{j}+g^{j j} \partial_{j}^{2}+\frac{1}{4} g_{, j, j}^{j j}-\right. & \left.\frac{1}{16} g_{j j}\left(g_{, j}^{j j}\right)^{2}\right) \Psi \\
& =(E-V) \Psi
\end{aligned}
$$

in which $g^{j j}=\partial_{j} g^{j j}$ and $g_{, j, j}^{j j}=\partial_{j} \partial_{j} g^{j j}$. Our formalism also addresses the very recent work of Chung and Hassanabadi [12], where the three-dimensional quantum mechanics in a curved space, based on the $q$-addition, is studied. By looking at the Eqs. (16)-(23) in Ref. [12], one observes that their momentum operator suffers from the lack of Hermiticity (see the disscussion after Eq. (78) of Ref. [13] ). This can be seen from the definition of the $q$-momentum operator in Eq. (10) of the Ref. [12] where $\hat{p}=-i \hbar D_{x}$ or equivalently

$$
\hat{p}=-i \hbar \frac{1}{1+q x} \partial_{x} .
$$

In terms of the canonical momentum, i.e., $\hat{p}_{0}=-i \hbar \partial_{x}$, it may be written as

$$
\hat{p}=\frac{1}{1+q x} \hat{p}_{0}
$$

Knowing that the canonical momentum is Hermitian, with respect to the standard definition, one finds

$$
\hat{p^{\dagger}}=\hat{p}_{0} \frac{1}{1+q x}=i \hbar \frac{q}{(1+q x)^{2}}+\hat{p}
$$


which is not clearly Hermitian i.e., $\hat{p}^{\dagger} \neq \hat{p}$ unless $q=0$. In terms of the line element given in Ref. [12] (in Eqs. (20) and (21)), the corresponding Schrodinger equation (19) reads

$$
\begin{array}{r}
-\frac{\hbar^{2}}{2 m} \sum_{j=1}^{3}\left(\left(1+q x_{j}\right)^{2} \partial_{j}^{2}+2 q\left(1+q x_{j}\right) \partial_{j}+\frac{1}{4} q^{2}\right) \Psi \\
=(E-V) \Psi
\end{array}
$$

(Note that here we use the convention $x_{j}=x, y, z$ for $j=$ $1,2,3$, respectively, while $\partial_{j}$ stands for $\frac{\partial}{\partial x_{j}}$.) Continuing with the particle in a three-dimensional infinite box where

$$
V(x, y, z)=\left\{\begin{array}{ll}
0 & 0<x, y, z<L \\
\infty & \text { elsewhere }
\end{array},\right.
$$

the wave function is obtained to be

$$
\begin{array}{r}
\Psi(x, y, z)=\frac{B}{\sqrt{(1+q x)(1+q y)(1+q z)}} \\
\times \sin \left[\frac{\ln (1+q x)}{\ln (1+q L)} n_{x} \pi\right] \sin \left[\frac{\ln (1+q y)}{\ln (1+q L)} n_{y} \pi\right] \\
\times \sin \left[\frac{\ln (1+q z)}{\ln (1+q L)} n_{z} \pi\right],
\end{array}
$$

with the same energy spectrum given in Eq. (29) of Ref. [12] and $B$ as a normalization constant. The nontrivial consequence of the choice of $\hat{P}_{j}$ can be seen by comparing the eigenfunction (25) with (26) of the Ref. [12]. Without giving the details, one predicts the occurrence of similar differences for the other examples in Ref. [12].

Here in this paper, parallel to the seminal work of Filho et al. [11], we first commence our work with the modification of the quantum operators in terms of a background metric. When such credible modification is found, we extend the metric-dependent Schrodinger equation and Ehrenfest theorem. Going further, we will interpret our results and then try to expand our findings in terms of the Poincare half-plane metric for a free particle.

\section{MODIFICATION OF QUANTUM OPERATORS IN A CURVED SPACE}

To start our pursuit, we note that the internal product of two quantum states on a Riemannian manifold can be written in the same way as on the Euclidean geometry with a modified volume element that includes the imprint of the background metric. We then write the internal product of two quantum states, $\left|\chi\left(x_{1}, x_{2}, x_{3}\right)\right\rangle$ and $\left|\theta\left(x_{1}, x_{2}, x_{3}\right)\right\rangle$, in a non-Euclidean geometry as

$$
\langle\chi \mid \theta\rangle=\int \chi^{*}\left(x_{1}, x_{2}, x_{3}\right) \theta\left(x_{1}, x_{2}, x_{3}\right) \sqrt{|g|} d x_{1} d x_{2} d x_{3},
$$

in which $x_{1}, x_{2}$, and $x_{3}$ are the local coordinates, and the line element of the background space is introduced by $d s^{2}=g_{j k} d x^{j} d x^{k}$, where $j, k=1,2,3$. Furthermore, $|g|$ is the absolute value of the determinant of the metric tensor $g_{j k}$. For a diagonal background metric, the modified differential element $d x_{j}$ can be written as $g_{j j}^{-\frac{1}{2}} d x_{j}$ (no Einstein summation rule) with $g_{j j}$ being the diagonal elements of the background metric. Accordingly, the metric-dependent translation operator can be written as $[12]$

$$
T\left(g_{j j}^{-\frac{1}{2}}\left(x_{1}, x_{2}, x_{3}\right) d x^{j}\right)=1-\frac{i g_{j j}^{-\frac{1}{2}} p_{j} d x^{j}}{\hbar},
$$

and then

$$
T(\vec{x})=\exp \left(-\frac{i \vec{P} \cdot \vec{x}}{\hbar}\right)
$$

In the above relation, $\vec{P}$ is the modified momentum operator in the presence of a non-flat diagonal background metric. This new operator leads to the modified commutation relation

$$
\left[x_{j}, P_{k}\right]=i \hbar g_{k k}^{-\frac{1}{2}}\left(x_{1}, x_{2}, x_{3}\right) \delta_{j k},
$$

which clearly depends on the geometry. In other words, this commutation is position-dependent and requires the modification of momentum operator in the Euclidean space. The form of this altered momentum operator could be naively considered as $P_{j}=-i \hbar g_{j j}^{-\frac{1}{2}} \partial_{j}$. However, this form does not satisfy the Hermiticity of the new non-flat momentum operator and thus needs to be modified as in (17). Note that, this Hermitian modified momentum operator obeys the same commutation relation in (29) since the augmented term $\partial_{j} g_{j j}^{-\frac{1}{2}}$ is only position-dependent and thus does not change the aforementioned commutation relation. Now, we proceed with the injection of this new Hermitian geometric momentum operator into the fundamental form-invariant quantum equations. In this regard, the fact that the fundamental equations of physics must be form-invariant under the change of the canonical coordinates (due to the principle of Covariance [14]), must be respected. Hence, the Hamiltonian function for a particle with mass $m$ that moves in a non-Euclidean space can be expanded with the new canonical variables $x_{j}$ and $P_{j}$ as

$$
H=\frac{1}{2 m} \sum_{j} P_{j}^{2}+V(\vec{x})
$$

where one can expand $P_{j}^{2}$ operator with (17) to obtain

$$
P_{j}^{2}=-\frac{\hbar^{2}}{4}\left[4 g_{, j}^{j j} \partial_{j}+g_{, j, j}^{j j}+4 g^{j j} \partial_{j}^{2}-\frac{1}{4} g_{j j}\left(g_{, j}^{j j}\right)^{2}\right]
$$


By inserting (31) into (30), the following relation for the three-dimensional modified Hamiltonian is obtained which can be cast into the form (19)

$$
\begin{aligned}
H=-\frac{\hbar^{2}}{2 m} \sum_{j=1}^{3}\left[g^{j j} \partial_{j}^{2}\right. & +g_{, j}^{j j} \partial_{j}+\frac{1}{4} g_{, j, j}^{j j} \\
& \left.-\frac{1}{16} g_{j j}\left(g_{, j}^{j j}\right)^{2}\right]+V(\vec{x}) .
\end{aligned}
$$

Note that, all the terms of the Hamiltonian in a curved space are dependent on the background metric in the absence of any imposed potential, say for a free particle. Moreover, the Hermiticity of the modified momentum operator, introduced by the last two terms in the brackets, is position-dependent. In consequence, the commutation of the Hermitian modified momentum operator with the Hamiltonian differs from the non-Hermitian form of the modified momentum operator introduced before. This leads to new terms in the Heisenberg equation for the new Hermitian canonical coordinate $x_{j}$. In this regard, we explicitly see that the Hermiticity condition of the modified momentum operator must be granted so one can find a reliable behavior in a curved space.

\section{EHRENFEST THEOREM IN A CURVED SPACE}

Imposing quantum mechanics on a curved space may add implications that are worth probing. One of these implications is the additional term in the Heisenberg uncertainty principle by which a non-zero minimal momentum would arise [15-17]. In fact, if a background metric is a smooth function of the class $C^{\infty}$, derivatives of the metric from any order exist and are continuous. Thus, Taylor-series expansion of this metric leads to the emergence of a quadratic term $x_{j}^{2}$ in the Heisenberg uncertainty principle, if the expansion is truncated at the second order. Consequently, this modification will cause a non-zero minimal energy in any quantum system that is being studied in a curved space. Looking more carefully, the emergence of a non-zero minimal energy for a quantum system in a curved space is consistent with the notion of general relativity that implies the connection between energy-momentum tensor and metric of the space. Furthermore, this modification underlines the non-commutativity and discretization of the momentum space [15]. This can be expressed as

$$
\Delta x_{j} \Delta P_{k} \geq \frac{\hbar}{2}\left[1+\alpha\left(\Delta x_{k}^{2}+\left\langle x_{k}\right\rangle^{2}\right)\right] \delta_{j k},
$$

in which $\alpha$ is a positive arbitrary constant that causes significant modifications in the very large scales. In the line of the descriptions above, $\alpha$ would be relevant to the emergent minimal energy of a quantum system. This can be easily interpreted for the case $\left\langle x_{k}\right\rangle=0$, by choosing the appropriate coordinate system, as writing Eq. (33) in the equality limit. In this way, the minimal measurable momentum is calculated as $\hbar \sqrt{\alpha}$, implying the physical meaning of $\alpha$ in the Eq. (33). There have been so far some theoretical efforts to constrain the value of $\alpha$ in the literature [18], but none of them could be well linked to the phenomenology in an experimental manner.

The modified momentum operator in a curved space can be further investigated in the Heisenberg picture. One may try to examine any likely revamp of the Ehrenfest theorem in a curved space assuming that the Heisenberg equation is still valid in a non-flat space. For a particle with mass $m$, it is found that

$$
\frac{d\left\langle x_{j}\right\rangle}{d t}=\frac{1}{i \hbar}\left\langle\left[x_{j}, H\right]\right\rangle=\frac{1}{2 m}\left\langle\left\{g_{j j}^{-\frac{1}{2}}, P_{j}\right\}\right\rangle,
$$

and

$$
\frac{d\left\langle P_{k}\right\rangle}{d t}=\frac{1}{i \hbar}\left\langle\left[P_{k}, H\right]\right\rangle=-\left\langle g_{k k}^{-\frac{1}{2}} \partial_{k} V(\vec{x})\right\rangle,
$$

where \{\} denotes anti-commutation. Mathematical manipulation of (35) leads to another useful relation given by

$$
\frac{d\left\langle P_{k}\right\rangle}{d t}=\frac{1}{2}\left\langle g_{k k}^{-\frac{1}{2}} \frac{d}{d t}\left\{g_{k k}^{-\frac{1}{2}}, P_{k}\right\}\right\rangle .
$$

Using Eqs. (35) and (36), one can write the relation

$$
\begin{aligned}
& \frac{d\left\langle P_{k}\right\rangle}{d t}= \\
& \quad-\frac{1}{2}\left\langle g_{k k}^{-\frac{1}{2}} \partial_{k} V(\vec{x})\right\rangle+\frac{1}{4} \frac{d}{d t}\left\langle g_{k k}^{-\frac{1}{2}}\left\{g_{k k}^{-\frac{1}{2}}, P_{k}\right\}\right\rangle
\end{aligned}
$$

in the Heisenberg picture. We would like to add that a specific form of the latter relation has been found in [11] (Eq. (11)) where $\left\langle p_{x}\right\rangle$, in the lhs, stands for $m\langle\dot{x}\rangle$. The term $g_{k k}^{-\frac{1}{2}}\left\{g_{k k}^{-\frac{1}{2}}, P_{k}\right\}$ can be further simplified using

$$
\left[P_{k}, g_{k k}^{-\frac{1}{2}}\right]=-i \hbar g_{k k}^{-\frac{1}{2}} g_{k k, k}^{-\frac{1}{2}}
$$

to obtain

$$
g_{k k}^{-\frac{1}{2}}\left\{g_{k k}^{-\frac{1}{2}}, P_{k}\right\}=2 g^{k k} P_{k}-\frac{i \hbar}{2} g_{k k}^{-\frac{1}{2}} g_{, k}^{k k},
$$

where the second term on the right-hand side depends only on the position. Finally, Eq. (37) can be expressed as

$$
\frac{d\left\langle P_{k}\right\rangle}{d t}=-\frac{1}{2}\left\langle g_{k k}^{-\frac{1}{2}} \partial_{k} V(\vec{x})\right\rangle+\frac{1}{2} \frac{d}{d t}\left\langle g^{k k} P_{k}\right\rangle,
$$

assuming that the background metric is static. The latter equation can be considered as the modified Ehrenfest theorem in a curved space. Evidently, the choice $g_{k k}=1$ 
reduces this equation to its original form in Euclidean space, given by

$$
\frac{d\left\langle P_{k}\right\rangle}{d t}=-\left\langle\partial_{k} V(\vec{x})\right\rangle
$$

It must be noted that the Ehrenfest theorem was acquired from the Heisenberg equation with the assumption that the Heisenberg equation is form-invariant in a curved space, comparing with a flat geometry. In consequence, it is reasonable to expect that the Ehrenfest theorem is form-invariant in a curved space, as well. As it was mentioned, Eq. (40) can be looked at as a modification of the Ehrenfest theorem in a curved space. However, the same flat form for the Ehrenfest theorem can be deduced considering a new geometric position-dependent differential operator $D_{k} \equiv \frac{1}{2} g_{k k}^{-\frac{1}{2}} \partial_{k}$, along with an effective potential that includes the imprint of the curved space [19]. This effective potential comprises an external imposed potential as well as tidal forces due to the background geometry. Thus, for a static background metric in the Heisenberg picture, Eq. (40) can be written in the form

$$
\frac{d\left\langle P_{k}\right\rangle}{d t}=-\left\langle D_{k}\left(V_{\text {ext }}(\vec{x})+V_{\text {curv }}(\vec{x})\right)\right\rangle,
$$

in which $D_{k} V_{\text {curv }}(\vec{x}) \equiv-\frac{1}{2} \frac{d}{d t}\left(g^{k k} P_{k}\right)$. In this fashion, the invariant form of the Ehrenfest theorem in a curved space with the modified differential operator $D_{k}$ and the mapped effective potential $V_{\text {eff }}(\vec{x}) \equiv V_{\text {ext }}(\vec{x})+$ $V_{\text {curv }}(\vec{x})$ can be written as

$$
\frac{d\left\langle P_{k}\right\rangle}{d t}=-\left\langle D_{k} V_{\text {eff }}(\vec{x})\right\rangle
$$

For instance, we may consider a free particle on which no external potential is imposed. Thus, Eq. (40) reads

$$
\frac{d\left\langle P_{k}\right\rangle}{d t}=\frac{1}{2} \frac{d}{d t}\left\langle g^{k k} P_{k}\right\rangle
$$

that non-trivially indicates a non-zero average force acting on a free particle due to the curved space. From the above discussion, it is obvious that the average force imposed on the free particle vanishes when the background geometry is flat. Hence, no quantum particle can be considered free from exterior potentials in a curved space, contrary to the Euclidean case. It is interesting to note that the geometry of the space does not impose any average force on a quantum system in case of a flat geometry. This implication can be concluded from Eq. (44), where a flat metric results in

$$
\frac{d\left\langle P_{k}\right\rangle}{d t}=0 .
$$

This outcome explicitly shows that only a spatially changing geometry of the space is able to impose an effective average force on a quantum system in view of the modification of quantum mechanics in a curved space.

\section{A FREE PARTICLE ON THE POINCARE HALF-PLANE GEOMETRY}

We proceed with investigating the behavior of a quantum particle free from any external potentials in the Poincare upper half-plane geometry. This represents a two-dimensional hyperbolic upper half-plane geometry [20] that imposes an effective constraining force on the particle, based on our above-mentioned results. The metric for the upper half-plane $x$ is defined as

$$
d s^{2}=\frac{d x^{2}+d y^{2}}{x^{2}}, \quad x>0,
$$

which has no dependence on dimension $z$. Hence, the wave function of the particle will be independent of $z$ according to Eq. (8), considering $V(x, y, z)=0$.

The modified time-independent Schrodinger equation $H \Psi(x, y)=E \Psi(x, y)$ in the new geometry is explicitly given by

$$
\begin{aligned}
& -\frac{\hbar^{2}}{2 m}\left[x^{2}\left(\partial_{x}^{2} \Psi(x, y)+\partial_{y}^{2} \Psi(x, y)\right)\right. \\
& \left.+2 x \partial_{x} \Psi(x, y)+\frac{1}{4} \Psi(x, y)\right]=E \Psi(x, y), \quad x>0
\end{aligned}
$$

in accordance with the Hamiltonian (8) and the line element (46). To solve Eq. (47), we assume that $\Psi(x, y)$ is a separable function with respect to its arguments, i.e. $\Psi(x, y)=\phi(x) \theta(y)$, noting the commutativity of the Poincare geometry. Thus Eq. (47) reads as

$$
\begin{aligned}
-\frac{\hbar^{2}}{2 m}[ & \frac{1}{\phi(x)} \frac{d^{2} \phi(x)}{d x^{2}}+\frac{2}{x \phi(x)} \frac{d \phi(x)}{d x} \\
& \left.+\frac{1}{4 x^{2}}+\frac{1}{\theta(y)} \frac{d^{2} \theta(y)}{d y^{2}}\right]=\frac{E}{x^{2}} \quad, x>0,
\end{aligned}
$$

which leads to separate equations in the set

$$
\left\{\begin{array}{l}
\frac{1}{\phi(x)} \frac{d^{2} \phi(x)}{d x^{2}}+\frac{2}{x \phi(x)} \frac{d \phi(x)}{d x}+\frac{\frac{1}{4}+\frac{2 m E}{\hbar^{2}}}{x^{2}}=A \\
\frac{1}{\theta(y)} \frac{d^{2} \theta(y)}{d y^{2}}=-A
\end{array},\right.
$$

for $x>0$ with $A$ being an arbitrary constant. The latter equation for $\theta(y)$ is similar to its counterpart for a free particle in the Euclidean space and has the solution

$$
\theta(y)=C_{1} e^{i k y}+C_{2} e^{-i k y},
$$

where $k \equiv \sqrt{A}$ is the spatial frequency of the particle, while $C_{1}$ and $C_{2}$ are integration constants. The solution to the first equation in (49) is of type Bessel function. To 
find the exact solution, one can transform it into a standard Schrodinger equation by a point canonical transformation [21] (and references therein). To this end, we apply the transformation

$$
\phi(x)=\frac{1}{\sqrt{x}} \zeta(z(x)),
$$

where

$$
z(x)=\ln (x)
$$

to obtain

$$
-\frac{\hbar^{2}}{2 m}\left(\frac{d^{2} \zeta(z)}{d z^{2}}\right)+V_{\mathrm{eff}}(z) \zeta(z)=E \zeta(z),
$$

with

$$
V_{\text {eff }}(z)=\frac{k^{2} \hbar^{2}}{2 m} e^{2 z}
$$

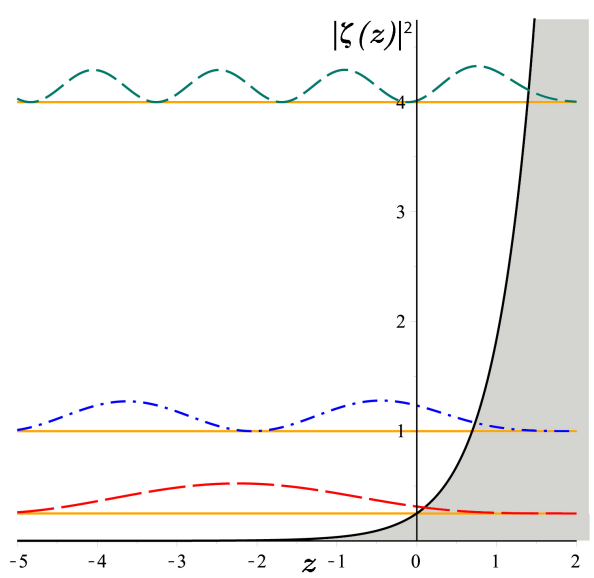

FIG. 1. Non-normalized $|\zeta(z)|^{2}$ in terms of $z$ for $\omega=\frac{1}{2}$ (RedLong Dash), 1 (Blue-Dash Dot) and 2 (Green-Dash) with $k=\frac{1}{2}$. The scaled effective potential i.e., $V_{\text {eff }}(z) / \frac{k^{2} \hbar^{2}}{2 m}$ is also plotted in the solid (black) curve. The plots clearly depict the expected asymptotic behaviors when $z \rightarrow \pm \infty$. The horizontal lines stand for the scaled energy i.e. $\omega^{2} \equiv \frac{2 m E}{\hbar^{2}}$.

Introducing $\omega^{2} \equiv \frac{2 m E}{\hbar^{2}}$, one finds a solution for the Schrodinger equation (53), in terms of the modified Bessel functions $I_{\nu}$ and $K_{\nu}$ of the first and the second kind, respectively. This solution is given by

$$
\zeta(z)=D_{1} I_{i \omega}\left(k e^{z}\right)+D_{2} K_{i \omega}\left(k e^{z}\right)
$$

in which $D_{1}$ and $D_{2}$ are integration constants. Due to the asymptotic behavior of the effective potential, $V_{\text {eff }}(z) \sim$ $e^{2 z}$, one expects the solution to be of the form of a plane wave for $z \rightarrow-\infty$, and zero when $z \rightarrow \infty$. Hence, one must set $D_{1}=0$ which implies

$$
\zeta(z) \sim K_{i \omega}\left(k e^{z}\right)
$$

with a continuous positive energy. In FIG. 1 we plot the non-normalized $|\zeta(z)|^{2}$ in terms of $z$ for $\omega=\frac{1}{2}, 1$ and 2 and $k=\frac{1}{2}$. The scaled effective potential and energy are also displayed.
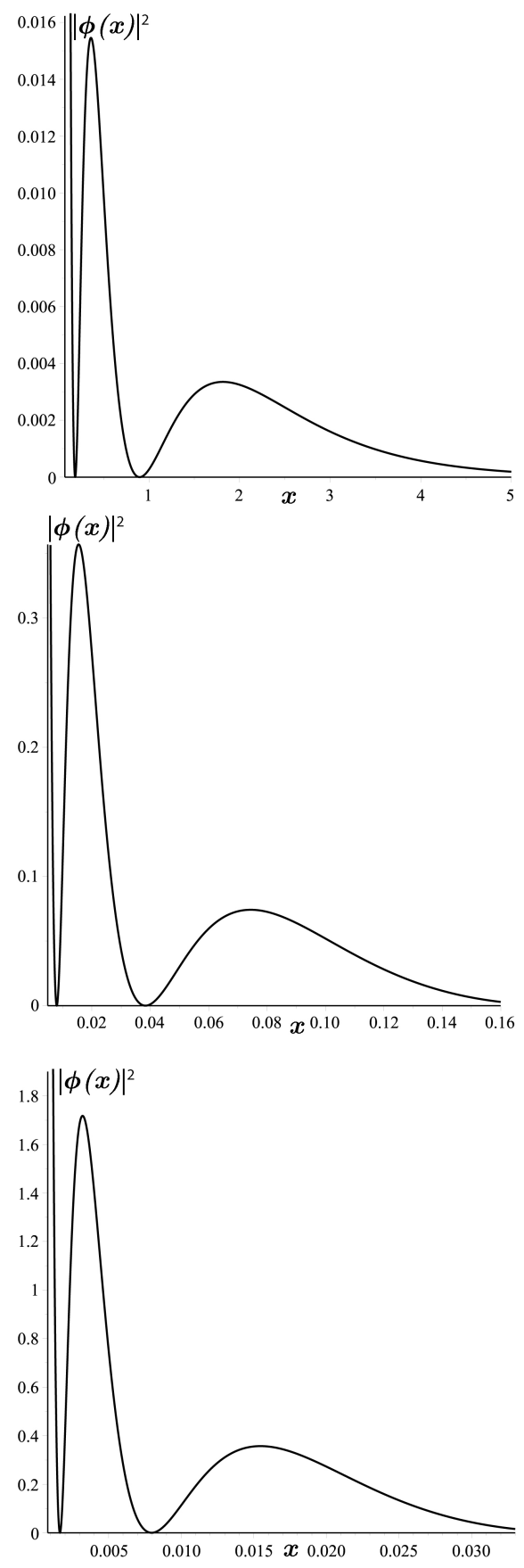

FIG. 2. Plots of $|\phi(x)|^{2}$ in terms of $x$ for $\omega=2$ and $k=\frac{1}{2}$ for three different intervals. The self-similarity of the probability densities is in agreement with the classical geodesics of the Poincare upper half-plane geometry (see the Appendix A).

The wave function in $x$ direction i.e., $\phi(x)$ is obtained 
through an inverse transformation and expressed as

$$
\phi(x) \sim \frac{1}{\sqrt{x}} K_{i \omega}(k x)
$$

up to a normalization constant. In Fig. 2 we plot $|\phi(x)|^{2}$ in terms of $x$ for $\omega=2$ and $k=\frac{1}{2}$ for three different intervals of $x$. This figure implies the self-similarity symmetry of the probability density for $x>0$. This is in agreement with the classical geodesics of the Poincare upper halfplane geometry which is scale-invariant / self-similar (see the Appendix A) .

We are also interested to inspect the energy spectrum in the case of very low effective potential, where $x$ tends to zero. This is being done for the sake of finding any clue for the emergence of non-zero minimal energy in the spectrum of a free particle, as placed on the upper halfplane geometry. In this way, we note that the second derivative of $\zeta(z)$ becomes zero in the mentioned limit, while the $\zeta(z)$ itself becomes indefinite. We then write Eq. (53) in the following form

$$
-\frac{\hbar^{2}}{2 m}\left(\frac{d^{2} \zeta(z)}{d z^{2}}\right)=\left(E-V_{\mathrm{eff}}\right) \zeta(z),
$$

and interpret that the term $E-V_{\text {eff }}$ must be equal to zero in the limit $x \rightarrow 0$. So, energy would be equal to the effective potential in the limit that the particle is asymptotically behaving as a plane wave. In advance, a minimal effective potential due to the curvature of the background, look at Eq. (44), and also due to accepting the minimal length cutoff (so that $|z|$ marks a maximum value in our case), can be regarded. In this manner we have shown that the claim of a minimal non-zero energy for a free quantum particle which is placed on the upper half-plane background can be confirmed.

\section{CONCLUSION}

To migrate from the quantum mechanics on the $\mathrm{Eu}-$ clidean space to its extension on a curved space, one should include the metric-dependent terms in the relevant generalized equations. In this regard, our geometrical approach in this study was initiated by designating a modified momentum operator such that it conveys the geometry of a curved space while engrossing Hermiticity. By this definition, the extraction of the corresponding Hamiltonian and the interpretation of the generalized Ehrenfest theorem followed. It was observed that Hamiltonian depends on the components of the background curved space, as well as the position of the particle. This, in turn, leads to emergence of new terms in the Heisenberg equation and modifies the Ehrenfest theorem (Eq. 40). However, it was shown that the Ehrenfest theorem could be put in an invariant form using a new geometric position-dependent differential operator $D_{k} \equiv \frac{1}{2} g_{k k}^{-\frac{1}{2}} \partial_{k}$ and an effective potential that includes the tidal forces due to the curved background geometry. More importantly, it can be understood from (44) that flat space imposes no constraint on a free particle, although it is not true for a general geometry.

In continuation, we derived the wave function of a "free particle" moving in the Poincare upper half-plane geometry. It was observed that the "free particle" that is indeed free along the $y$-axis, actually behaves as if it is bounded by the curved space along the $x$-axis (FIG. 1), due to the term $\frac{1}{\sqrt{x}}$ in $\phi(x)$. In fact, there exists an effective potential barrier that surges exponentially towards infinity as $x \rightarrow+\infty$. Thus, the probability density $|\phi(x)|^{2}$ approaches zero while $x$ increases towards infinity. This, of course, is explicable due to the Poincare half-plane geometry, for which the geodesics of a classical free particle are also bounded. In addition, we should notice the behavior of the particle while $x \rightarrow 0$. In FIG. 2, the probability density shows a fractal character in the vicinity of $x=0$, added by its increase while moving towards $x=0$. In fact, effective potential becomes zero while $x \rightarrow 0$. In consequence, we expect the oscillatory behavior of a Euclidean free particle as it is but with the increasing probability density as $x \rightarrow 0$. To recapitulate, a particle free from any exterior potential that is put in the Poincare upper half-plane geometry experiences a bounded behavior in accord with its classical fractal geodesics.
[1] C.C. Barros, Eur. Phys. J. C 42, 119 (2005). I, I

[2] W.S. Chung and H. Hassanabadi, Mod. Phys. Lett. A 32, 26 (2017).

[3] W.S. Chung and H. Hassanabadi, Mod. Phys. Lett. A 33, 1850150 (2018).

[4] M. Park, Phys. Lett. B 659, 698 (2008).

[5] T. V. Fityo, I. O. Vakarchuk and V. M. Tkachuk, J. Phys. A: Math. Theor. 41, 4 (2008).

[6] Kh. P. Gnatenko, arXiv:1909.06295.

[7] L. Perivolaropoulos, Phys. Rev. D 95, 103523 (2017).
[8] K. Nozari and P. Dehghani, Phys. Lett. B 792, 101 (2019).

[9] P. Dehghani and K. Nozari, arXiv:2002.10279. I

[10] B. Hamil and M. Merad, Eur. Phys. J. Plus 133, 174 (2018). I

[11] R.N.C. Filho, J.P.M. Braga, J.H.S. Lira and J.S. Andrade, Phys. Lett. B 755, 367 (2016) (document), I, I, III

[12] W.S. Chung and H. Hassanabadi, Int. J. Mod. Phys. A 34, 1950177 (2019). I, I, I, II 
[13] A. Lavagno, A. M. Scarfone and P. N. Swamy, Eur. Phys. J. C 47, 253 (2006). I

[14] S.K. Kauffmann, arXiv:1005.2641. II

[15] S. Ghosh and S. Mignemi, Int. J. Theor. Phys 50, 1803 (2011). III

[16] B. Hamil, M. Merad and T. Birkandan, Eur. Phys. J. Plus 134, 278 (2019).

[17] J.L.A. Coelho and R.L.P.G. Amaral, Phys. A: Math. Gen 35, 5255 (2002). III

[18] B. Mirza and M. Zarei, Phys. Rev. D 79, 125007 (2009). III

[19] I. V. Kanatchikov, J. Geom. Symmetry Phys. 37, 43 (2015). III

[20] A. Terras, Harmonic Analysis on Symmetric SpacesEuclidean Space, the Sphere, and the Poincare Upper Half-Plane (Springer, New York, 2013). IV

[21] K.H. Yeon, S.K. Hong, C.I. Um and T.F. George, Phys. Lett. A 357, 12 (2006).

IV

\section{Appendix A: Classical Geodesics in the Poincare Upper Half Plane}

Considering the line-element (46), the classical geodesics equation in the Poincare upper half-plane is given by

$$
\frac{d^{2} x^{i}}{d \sigma^{2}}+\Gamma_{j k}^{i} \frac{d x^{j}}{d \sigma} \frac{d x^{k}}{d \sigma}=0
$$

in which $\sigma$ is the geodesic parameter and $\Gamma_{j k}^{i}$ are the Christoffel symbols. Knowing that the only nonzero Christoffel symbols are $\Gamma_{x x}^{x}=-\Gamma_{y y}^{x}=-\Gamma_{x x}^{y}=\frac{1}{x}$, one finds the following equations

$$
\ddot{x}-\frac{1}{x} \dot{x}^{2}+\frac{1}{x} \dot{y}^{2}=0
$$

and

$$
\ddot{y}-\frac{2}{x} \dot{x} \dot{y}=0
$$

in which a dot stands for the derivative with respect to $\sigma$. Eq. (A3) yields

$$
\dot{y}=\frac{\dot{y}_{0}}{x_{0}^{2}} x^{2}
$$

and consequently Eq. (A2) admits

$$
\dot{x}=x\left(\frac{\dot{x_{0}}+\dot{y}_{0}}{x_{0}}-\frac{\dot{y_{0}}}{\dot{x}_{0}^{2}} y\right) .
$$

For, $\dot{y}_{0}=0$, the geodesic is a straight line parallel to the $x$-axis. With $\dot{y}_{0} \neq 0$, the combination of (A4) and (A5) gives

$$
\frac{d y}{d x}=\frac{\frac{\dot{y}_{0}}{x_{0}^{2}} x}{\left(\frac{\dot{x_{0}+\dot{y}_{0}}}{x_{0}}-\frac{\dot{y}_{0}}{\dot{x}_{0}^{2}} y\right)} .
$$

The latter first order ODE admits exact solution given by

$$
x^{2}+\left(y-y_{c}\right)^{2}=R_{c}^{2}
$$

which is the geodesic of the classical particle. Herein, $y_{c}=x_{0}\left(1+\frac{\dot{x}_{0}}{\dot{y}_{0}}\right)$ and $R_{c}^{2}=x_{0}^{2}+\left(y_{0}-y_{c}\right)^{2}$ and the curve is a semi-circle centered at $\left(0, y_{c}\right)$ with radius $R_{c}$. It is worth mentioning that, the geodesic in Poincare upper half plane is self-similar or scale-invariant. To see this let's introduce the transformation $x=\lambda \widetilde{x}$ and $y=\lambda \widetilde{y}$ for some scale factor $\lambda$. Upon this transformation, Eq. (A7) becomes

$$
\widetilde{x}^{2}+\left(\widetilde{y}-\frac{y_{c}}{\lambda}\right)^{2}=\frac{R_{c}^{2}}{\lambda^{2}}
$$

which is again a semi circle with scaled radius $\widetilde{R}_{c}$, centered at $\left(0, \widetilde{y}_{c}\right)$ in which $y_{c}=\lambda \widetilde{y}_{c}$ and $R_{c}=\lambda \widetilde{R}_{c}$ with the same scale factor. 